\title{
Maize brachytic2 (br2) suppresses the elongation of lower internodes for excessive auxin accumulation in the intercalary meristem region
}

Xiangge Zhang ${ }^{1,2+}$, Xianbin Hou ${ }^{3+}$, Yinghong Liu ${ }^{4}$, Lanjie Zheng ${ }^{2}$, Qiang Yi ${ }^{2}$, Haojun Zhang ${ }^{2}$, Xinrong Huang ${ }^{2}$, Junjie Zhang ${ }^{5}$, Yufeng Hu ${ }^{1,2}$, Guowu Yu ${ }^{2}$, Hanmei Liu ${ }^{5}$, Yangping Li ${ }^{1,2}$, Huanhuan Huang ${ }^{2}$, Feilong Zhan ${ }^{4}$, Lin Chen ${ }^{2}$, Jihua Tang ${ }^{6^{*}}$ and Yubi Huang ${ }^{1,2^{*}}$

\begin{abstract}
Background: Short internodes contribute to plant dwarfism, which is exceedingly beneficial for crop production. However, the underlying mechanisms of internode elongation are complicated and have been not fully understood.

Results: Here, we report a maize dwarf mutant, dwarf2014 (d2014), which displays shortened lower internodes. Map-based cloning revealed that the $\$ 2014$ gene is a novel br2 allele with a splicing variation, resulting in a higher expression of BR2-T02 instead of normal BR2-T01. Then, we found that the internode elongation in d2014/br2 exhibited a pattern of inhibition-normality-inhibition (transient for the ear-internode), correspondingly, at the 6-leaf, 12-leaf and 14-leaf stages. Indeed, BR2 encodes a P-glycoprotein1 (PGP1) protein that functions in auxin efflux, and our in situ hybridization assay showed that BR2 was mainly expressed in vascular bundles of the node and internode. Furthermore, significantly higher auxin concentration was detected in the stem apex of d2014 at the 6leaf stage and strictly in the node region for the ear-internode at the 14-leaf stage. In such context, we propose that BR2/PGP1 transports auxin from node to internode through the vascular bundles, and excessive auxin accumulation in the node (immediately next to the intercalary meristem) region suppresses internode elongation of d2014.
\end{abstract}

Conclusions: These findings suggest that low auxin levels mediated by BR2/PGP1 in the intercalary meristem region are crucial for internode elongation.

Keywords: Auxin efflux, br2, Dwarfism, Internode elongation, Intercalary meristem, Maize

\section{Background}

Dwarfism or semi-dwarfism confers a number of advantages to crop varieties, such as increased lodging resistance, denser growth, and higher harvest index, which are extraordinarily beneficial for crop production [1-3]. For crops, height is usually determined by the

\footnotetext{
* Correspondence: tangjihua1@163.com; yubihuang@sohu.com ${ }^{+}$Xiangge Zhang and Xianbin Hou contributed equally to this work. ${ }^{6}$ National Key Laboratory of Wheat and Maize Crop Science, Henan Agricultural University, Zhengzhou 450000, Henan, China

${ }^{1}$ State Key Laboratory of Crop Genetics of Disease Resistance and Disease Control, Sichuan Agricultural University, Chengdu 611130, Sichuan, China Full list of author information is available at the end of the article
}

internodes' number and length [4]. Numerous dwarf mutants have been characterized by short internodes, which have been attributed to impaired internode elongation. Therefore, increasing our knowledge of internode elongation will aid the improvement of crop yield.

Stem/internode elongation is mainly controlled by several plant hormones, including gibberellins (GAs), brassinosteroids (BRs), strigolactones (SLs), and auxins [5]. Defects in the biosynthesis or the signaling of these hormones can cause dwarf phenotypes [6-10], although each hormone might play a different role in stem/internode elongation, irregularities of which will lead to

(C) The Author(s). 2019 Open Access This article is distributed under the terms of the Creative Commons Attribution 4.0 International License (http://creativecommons.org/licenses/by/4.0/), which permits unrestricted use, distribution, and 
various mutant phenotypes. In maize, there are several GA-related mutants that display extremely reduced height with uniformed short internodes, such as dwarf1(d1), d3, d5 and anther ear1 (an1), as well as dominant mutants $D 8$ and $D 9$ [11-13], all of which influence internode elongation throughout the growth period, accompanied by a certain degree of yield loss. Most BR-associated mutants exhibit multiple defective phenotypes in addition to dwarfism [14-17]. For example, the maize nana plant2 (na2) mutant displays suppression of tillers, altered leaf morphology, and andromonoecy [18]. Unlike GAs and BRs, the reduction in plant height of SL mutants may be an indirect effect of increased tillers because the deficiency in SLs enhances cell division in axillary meristems (AMs) $[19,20]$, which ultimately leads to a redirection of the nutrition toward tiller growth instead of internode elongation.

Auxin biosynthesis is performed through multiple Trp-dependent or Trp-independent pathways, mainly involving YUCCA (YUC) and TAA1/TAR1/TAR2 genes [21]. Quadruple yuc1 yuc4 yuc10 yuc11 mutants do not develop a hypocotyl and root meristem [22]. Similarly, taa1 mutants in Arabidopsis displaye a defective hypocotyl and root [23] and taa1 tar1 tar2 triple mutants lacked roots and were seedling lethal [24]. In maize, the vanishing tassel2 (vt2) gene is an ortholog of Arabidopsis $T A A 1$, and $v t 2$ mutants exhibit no tassel branches or spikelets, as well as a semi-dwarf phenotype with fewer leaves [25]. Many organs, including the stem/internode, are damaged in auxin biosynthesis mutants. The $\mathrm{SCF}^{\mathrm{TIR} 1 / \mathrm{AFB}}$-mediated proteolysis of Aux/IAA proteins is the major auxin signaling pathway, which is clearly responsible for many auxin actions [26, 27]. Most mutants in these components have a similar seedling lethal phenotype [28-30]. In addition, synthesized auxin is often directionally transported by auxin transporters to specific tissues, where it acts a potent signal that triggers a plethora of developmental responses [31]. The maize $b r 2$ and sorghum orthologue dwarf3 (dw3) exhibit reduced auxin transport and shortened statures [32], and auxin transport is crucial for regulating internode elongation.

Actually, several dwarf mutants described above exhibited various abnormal patterns of internode elongation and other morphological characteristics, which suggests that their underlying mechanisms should be discriminating and be worth pursuing further. In this study, we identified a novel $b r 2$ allelic mutant, $d 2014$, that exhibited shortened lower internodes but nearly normal upper parts, indicating that $b r 2$ has a unique regulation on plant height development. The $b r 2$ is a very famous dwarf gene (first cloned in 2003), which was considered ideal for shortening maize's height due to its unique phenotype (mild dwarf, shorter lower internodes yet nearly normal upper internodes) [32, 33]. It was wellsuited to dissect the mechanism of plant height development for maize improvement. Here, in order to reveal the effects of the $d 2014 / b r 2$ mutation on internode elongation, we performed a dynamic comparison of internode elongation at several stages between $d 2014$ and wild type (WT) plant. Furthermore, we explored the specific location of $B R 2$ expression in the stem and detected the dynamic variation of auxin concentration so as to reveal the mechanism of internode elongation by BR2/PGP1-mediated auxin transport.

\section{Results}

\section{Characterization of the maize $\mathbf{d} 2014$ dwarf mutant}

A maize dwarf mutant, $d 2014$, arose spontaneously from the HL9047 (WT) inbred line in 2014, and its selfcrossed progenies steadily presented with uniform short stature with erect leaves (Fig. 1a). The plant height of $d 2014$ was reduced by $58.61 \mathrm{~cm}$, whereas its ear height was $44.59 \mathrm{~cm}$ less than that of WT (Table 1). This showed that the reduced height of lower internodes mainly contributes to the $d 2014$ dwarf phenotype. Additionally, other traits of $d 2014$, such as ear length, ear width, ear weight, hundred-grain weight, total tassel length, tassel branch number, tasseling stage, and total leaf number were altered slightly in comparison with WT (Fig. 1b; Table 1). Especially for the ear traits, small variations were not enough to lead to yield loss. Therefore, the $d 2014$ mutant might be useful in maize breeding programs.

To ascertain the genetic basis of dwarfism for $d 2014$, three $\mathrm{F}_{2}$ and three $\mathrm{BC}_{1}$ populations derived from $d 2014 \times$ HL5038,$d 2014 \times$ HL5054 and $d 2014 \times$ F19 were generated. $\mathrm{F}_{2}$ and $\mathrm{BC}_{1}$ populations presented tall or short individual separation. Through $\chi^{2}$ tests, the plant height segregated in a 3:1 ratio (tall/short) in all three $F_{2}$ populations and in a 1:1 ratio (tall/short) in three $\mathrm{BC}_{1}$ populations (Additional file 2: Table S2). These results indicated that this dwarf phenotype of $d 2014$ was genetically controlled by a single recessive gene.

\section{The $d 2014$ gene is a br2 allele}

To clone the dwarf $d 2014$ gene, we carried out positional cloning using the $(d 2014 \times \mathrm{HL} 5054) \times d 2014 \mathrm{BC}_{1}$ population. Firstly, $300 \mathrm{BC}_{1}$ individuals that had a similar dwarf phenotype as $d 2014$ were identified and genotyped by 150 pleomorphic simple sequence repeat (SSR) makers, and then the $d 2014$ gene locus was defined to a $40.05 \mathrm{Mb}$ genetic interval between marker umc1281 and umc1278 (Fig. 2a). Subsequently, 768 dwarf plants were genotyped for fine mapping by newly developed InDel molecular makers, and the $d 2014$ gene was narrowed to a smaller segment flagged by the two markers a4 and a15, which are $2.85 \mathrm{Mb}$ apart (Fig. 2a). Finally, 2000 

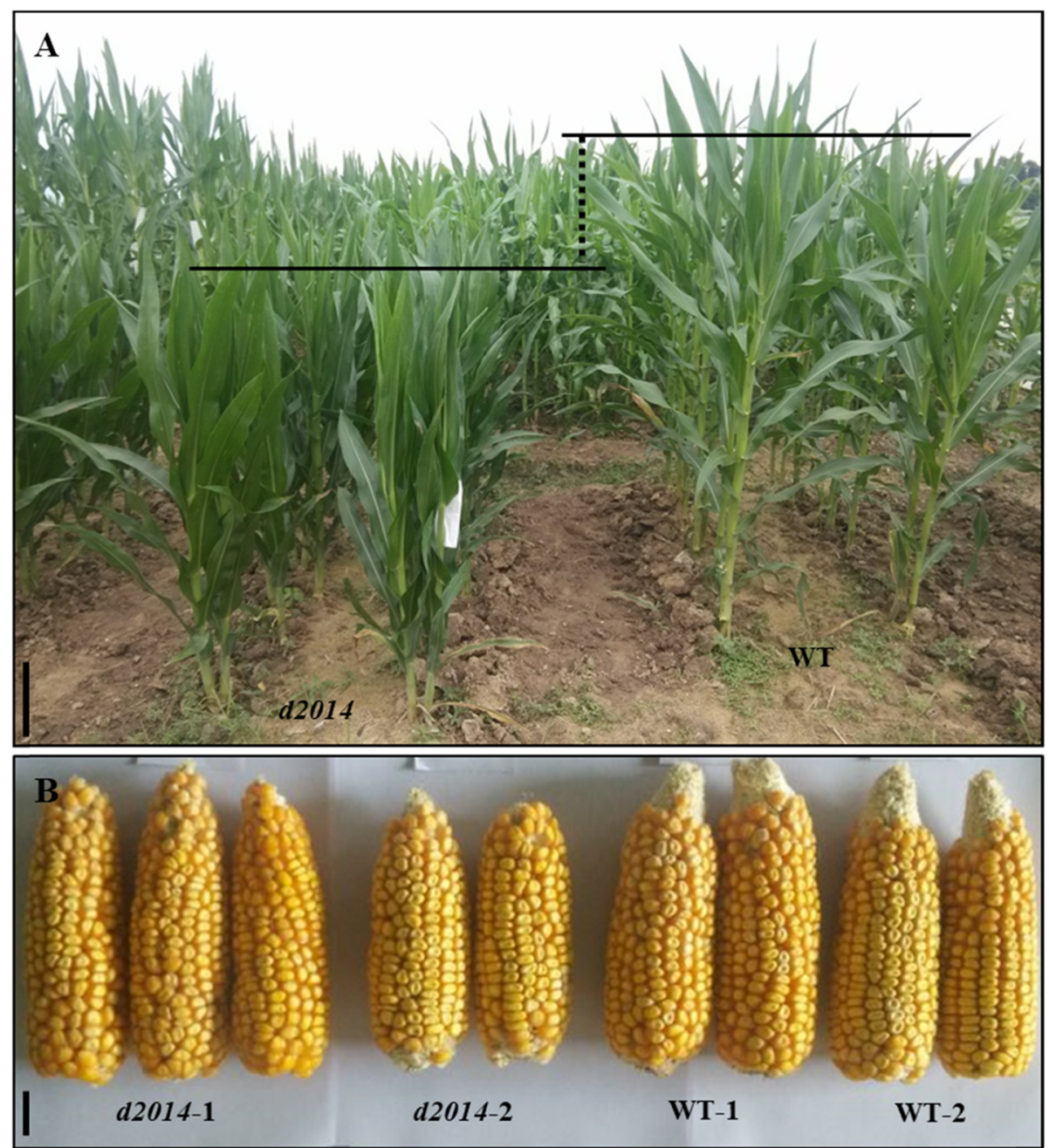

Fig. 1 Morphological comparison between d2014 and WT. a Plant morphology of d2014 and WT in the field at the heading stage. The vertical dotted line indicates the difference of plant height between d2014 and WT. Bar $=40 \mathrm{~cm}$. $\mathbf{b}$ Ear morphology of d2014 and WT at the mature stage. $B a r=2 \mathrm{~cm}$

recessive individuals were used to determine the candidate region of about $510 \mathrm{~Kb}$ that contained the $d 2014$ gene (Fig. 2a).

Database analysis showed that BR2 and six other candidate genes are within this $470 \mathrm{~Kb}$ region (http:// ensembl.gramene.org/biomart/martview/; Fig. 2b). Genomic DNA sequences of these genes from the $d 2014$ mutant and WT were compared, and the results showed that only the $B R 2$ sequences in $d 2014$ had two variant regions, including a $546 \mathrm{bp}$ insertion in intron $3\left(\mathrm{C}_{2073}\right)$ and a 233 bp deletion $\left(\mathrm{A}_{6568}\right.$ to $\left.\mathrm{C}_{6800}\right)$ in exon 5 (Fig. $2 \mathrm{~b})$. The inserted sequence in intron 3 seemed not to be characterized as transposable elements, while the missing region in exon 5 would result in the deletion of 92 amino acids and a frameshift affecting the translation of 29 additional amino acids (Fig. 2c). Moreover, a known br 2 mutant (114F) was crossed with $d 2014$ and WT, respectively. Significant differences in plant growth and development between the two hybrids were observed. At the 14-leaf stage, the morphological height of hybrid $114 \mathrm{~F} \times d 2014$ was significantly lower than that of hybrid $114 \mathrm{~F} \times \mathrm{WT}$, and the internode length of the former was strongly shortened in comparison with hybrid $114 \mathrm{~F} \times$ WT (Additional file 3: Figure S1). Over all, these results confirmed that the $d 2014$ gene was a $b r 2$ allele with a unique variation.

\section{The $d 2014 / b r 2$ mutation alters the normal splicing of $B R 2$} gene

Generally, a genetic mutation would cause the variation of natural expression pattern or protein function, resulting in phenotypic defects. Database analysis showed that BR2 had two transcripts: BR2-T01 (4672 bp) and BR2T02 (2061 bp) (https://www.maizegdb.org/). Actually, 
Table 1 Phenotypic comparison between d2014 and WT

\begin{tabular}{|c|c|c|c|}
\hline Traits & $d 2014$ & WT & Variation \\
\hline Plant height $(\mathrm{cm})$ & $163.02 \pm 2.00^{* * *}$ & $221.63 \pm 2.17$ & -58.61 \\
\hline Ear height $(\mathrm{cm})$ & $35.97 \pm 1.33^{* * *}$ & $80.56 \pm 0.67$ & -44.59 \\
\hline Total tassel length $(\mathrm{cm})$ & $45.50 \pm 5.67$ & $47.37 \pm 6.33$ & -1.87 \\
\hline Tassel branch number $(\mathrm{cm})$ & $11.67 \pm 2.07$ & $13.12 \pm 4.00$ & -1.45 \\
\hline Tasseling stage (day) & $50 \pm 0.32$ & $50 \pm 0.12$ & 0 \\
\hline Total leaf number & $20.49 \pm 0.45$ & $20.4 \pm 0.38$ & 0.09 \\
\hline Leaf number above the ear & $7.06 \pm 0.23$ & $6.70 \pm 0.27$ & 0.36 \\
\hline Ear length $(\mathrm{cm})$ & $14.13 \pm 0.49$ & $15.04 \pm 0.43$ & -0.91 \\
\hline Ear width $(\mathrm{cm})$ & $4.59 \pm 0.26$ & $5.16 \pm 0.20$ & -0.57 \\
\hline Grain length $(\mathrm{cm})$ & $1.09 \pm 0.02$ & $1.12 \pm 0.03$ & -0.03 \\
\hline Grain width $(\mathrm{cm})$ & $0.92 \pm 0.01$ & $0.89 \pm 0.02$ & 0.03 \\
\hline Ear weight (g) & $122.12 \pm 4.44$ & $133.25 \pm 4.89$ & -11.13 \\
\hline Hundred-grain weight (g) & $32.35 \pm 0.50$ & $32.23 \pm 0.43$ & 0.12 \\
\hline
\end{tabular}

Vaues indicate mean \pm SD. ${ }^{* * *}$ represents significant difference between $d 2014$ and WT at 0.001 level

BR2-T01 is composed of 5 exons, while BR2-TO2 only has 4 exons excluding the exon 5 (Fig. 3a). In addition to the 5'UTR, 3'UTR and exon 5, a vital region with an additional $15 \mathrm{bp}$ in BR2-TO2 exon 4 is different from that in BR2-T01 exon 4 (Fig. 3b). To verify the existence of these two transcripts in vivo, their cDNA sequences were amplified and compared. For BR2-T01, the band size of $d 2014$ was distinctly less than that of WT (Fig. $3 \mathrm{c}$ ), which was in agreement with a fragment deletion in $d 2014$. Sequencing the band revealed the previously predicted 233 bp deletion. Meanwhile, there was also a similar band of about $2000 \mathrm{bp}$ for BR2-T02 in d2014 and WT (Fig. 3c), the sequences of which were identical. This suggested that the two transcripts truly existed in vivo.

In view of this, it was necessary to analyze the effect of $d 2014 /$ br 2 mutation on the two transcripts. To define the expression patterns of BR2-T01 and BR2-T02, qRTPCR was carried out through specific quantitative primers. During the three stages of 6-leaf, 10-leaf and 15-leaf, the expression levels of BR2-T01 in d2014 were all lower than in WT, whereas BR2-T02 was significantly up-regulated in $d 2014$ during the three stages (Fig. $3 \mathrm{~d}$ ). These data suggested that the natural expression of $B R 2-$ T01 is crucial for WT normal growth, and the downregulated BR2-T01 leads to the defective traits of $d 2014$. Interestingly, the total $B R 2$ expression level was roughly similar between $d 2014$ and WT (Fig. 3d). These results revealed that the $d 2014$ mutation alters the normal splicing of $B R 2$ gene, causing a higher expression of $B R 2$ TO2 instead of normal BR2-T01.

\section{Analysis of BR2/PGP1 and auxin efflux in yeast}

In maize, BR2 encodes an auxin transporter, PGP1, which functions in auxin export from intercalary meristems [34]. In order to investigate whether the protein BR2/PGP1 (T01 and/or T02) is responsible for auxin transport at the cellular level, we functionally expressed these two variants in yeast. Fluorinated indole derivate (5-FI), a toxic analog of indole-3-acetic acid (IAA, namely auxin), is cytotoxic to yeast and has been used to investigate auxin transport [35, 36]. Yeast mutant strain gef1 lacks endogenous chloride channel protein [37], which results in 5-FI accumulation in gef1 cytoplasm after the undissociated 5-FI molecules entering cells by passive diffusion, leading to gefl's hypersensitivity to 5-FI. Using the yeast mutant strain gef1, we found that both of WT-PGP1-T01 and d2014PGP1-T01 provided certain resistance against 5-FI compared to the vector control (Fig. 4a). The result indicated that the protein PGP1-T01 exported 5-FI to the outside of the yeast cell, namely, PGP1-T01 was responsible for auxin export. Interestingly, PGP1-T02 also exhibited slight but significant resistance against 5 -FI in the yeast mutant strain gef1 (Fig. 4b), indicating its functions in auxin export. In terms of alternative splicing, there are mainly two types: front mutation (T01 and T02, both defective) and back mutation (T01, defective; T02, normal). When the two proteins, PGP1-T01 and PGP1-T02, play similar roles in auxin's export, the second type of mutation would display a mild phenotype, which opens the possibility that there is a variation of $b r 2$ mutant with diverse defects.

\section{Defects of internode elongation in the $d 2014$ mutant}

A typical characteristic of $b r 2$ mutants is that dwarf stature derives from shortened lower internodes [32, 34]. Of the $d 2014 / b r 2$ mutant, the length of certain internodes below the ear was dramatically reduced; additionally, the ear-internode was also shortened, whereas the internodes above the ear were approximately normal (Fig. 5a). Meanwhile, we found that other br2 mutants, $b r 2-114 F$ and $b r 2-117 A$, also displayed an abnormal ear-internode phenotype similar to $d 2014$ (Fig. 5b). These data showed that the loss-of-function alleles of $b r 2$ specifically regulated the elongation of certain internodes.

Since the total leaf numbers and tasseling stage of WT and $d 2014$ (Table 1) are nearly the same, thus, their developmental phase should be paralleled. To clarify how the mutation of $b r 2$ affects internode elongation, we carried out a dynamic comparison of stem growth between $d 2014$ and WT. Plenty of individuals were planted and leaf numbers were labeled to ensure the same developmental stage. Before the 12-leaf stage, the 6th, 7th, 8th, 9th, 10th, and 11th internodes in $d 2014$ were scarcely elongated (Fig. 6a, b, c, d), and the stem height of all these internodes was significantly lower than that in WT (Additional file 4: Figure S2A). From the 12-leaf stage to the 14-leaf stage, the $11^{\text {th }}$, and 12th internodes in $d 2014$ grew rapidly compared to previous patterns, and their lengths increased (Fig. 6d, e). Moreover, the length of 


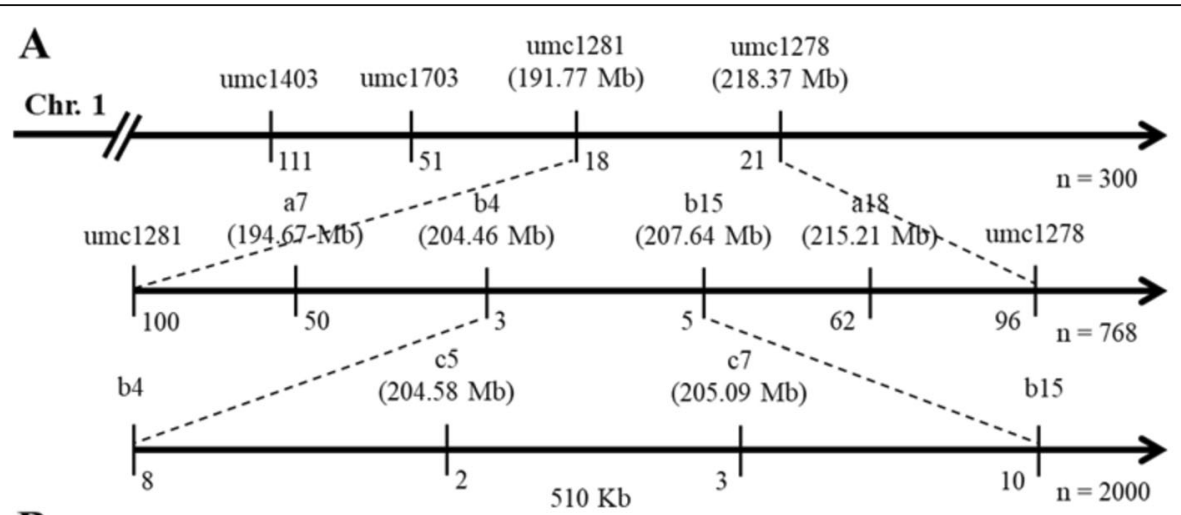

B

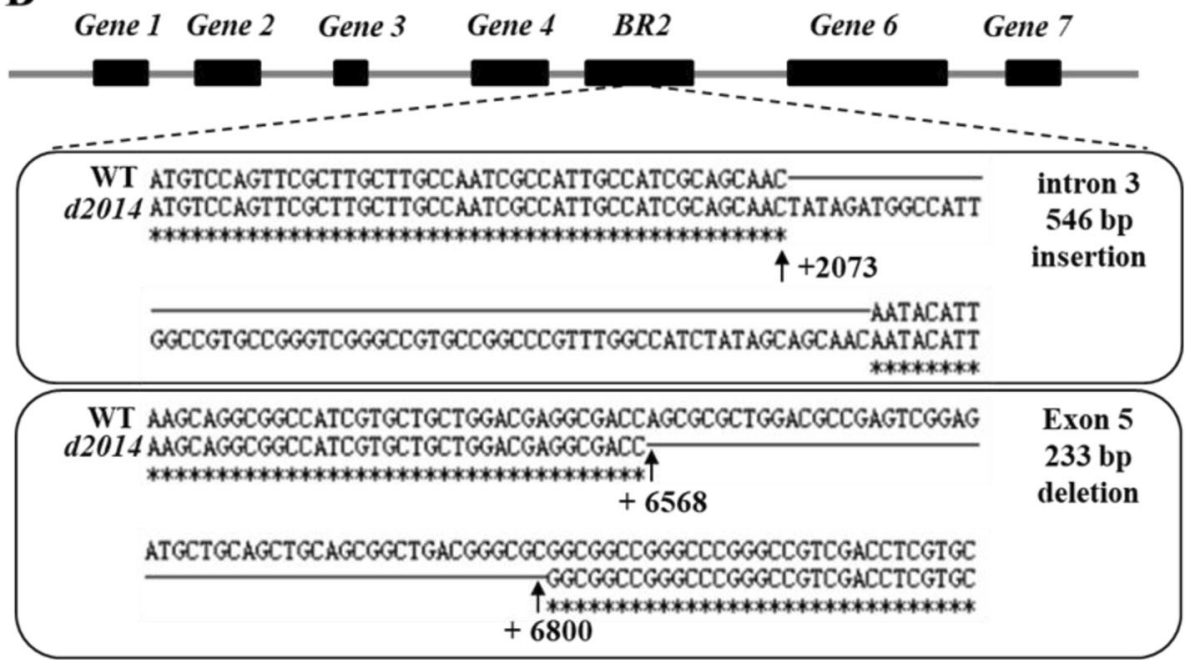

C
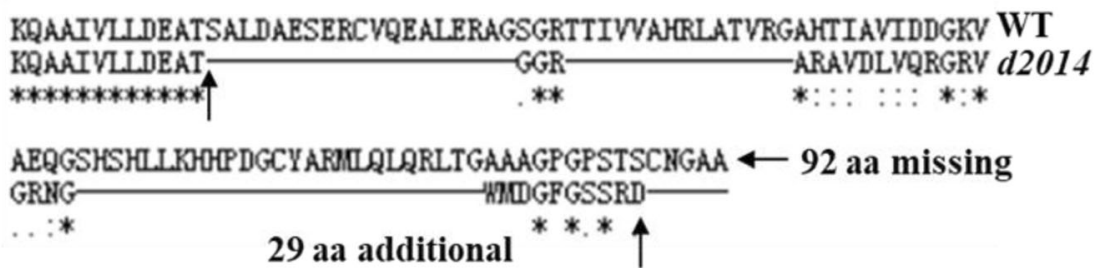

Fig. 2 Identification of the $d 2014$ gene. a Positional cloning of the d2014 gene: Firstly, the d2014 locus was mapped between the markers umc1281 and umc1278 on the chromosome 1 using 300 dwarf individuals from (d2014×HL5054) × d2014 BC1 population. Secondly, we enlarged the population and developed new Indel molecular markers. The location of the $d 2014$ gene was then narrowed down to a smaller segment flanked by the markers b4 and b15. Finally, using 2000 recessive plants, we locate the candidate region in about 470 Kb between the markers $\mathrm{c} 5$ and $\mathrm{C7}$. The numbers below the vertical lines indicate the recombinants. b The BR2 and other six predicted genes in the $470 \mathrm{~Kb}$ region, and and the sequence alignment of BR2 in d2014 and WT. c Variation of the BR2 protein sequence between d2014 and WT

the 11th, 12th and 13th internodes (ear-internode) in d2014 was similar to that of WT at the 14-leaf stage (Fig. 6e). Namely, the 5 lowest internodes in $d 2014$ were shortened seriously (Additional file 4: Figure S2B). Strangely, after the 14-leaf stage, the 11th, 12th and 13th internodes in $d 2014$ grew slowly in comparison with WT (Fig. 6e, f), and the elongation of the ear-internode was sharply restrained (Fig. $6 \mathrm{~g}$ ). Nevertheless, the patterns of upper internode elongation in both $d 2014$ and WT were exactly alike after the 16-leaf stage (Fig. 6g, h), which led to nearly paralleled length of the upper internodes (Additional file 4: Figure S2C). All these results revealed that defects of internode elongation in mutant $d 2014$ are disparate at different developmental stages; especially, at the 6-leaf, 12-leaf and 14-leaf stages, the internode elongation in $d 2014$ exhibits variation pattern of inhibited-normal-inhibited transiently.

Effect of the $d 2014$ mutation on cell morphology in shortened internodes

At an early developmental stage (before 12-leaf stage), the elongation of the lower internodes in $d 2014$ was 

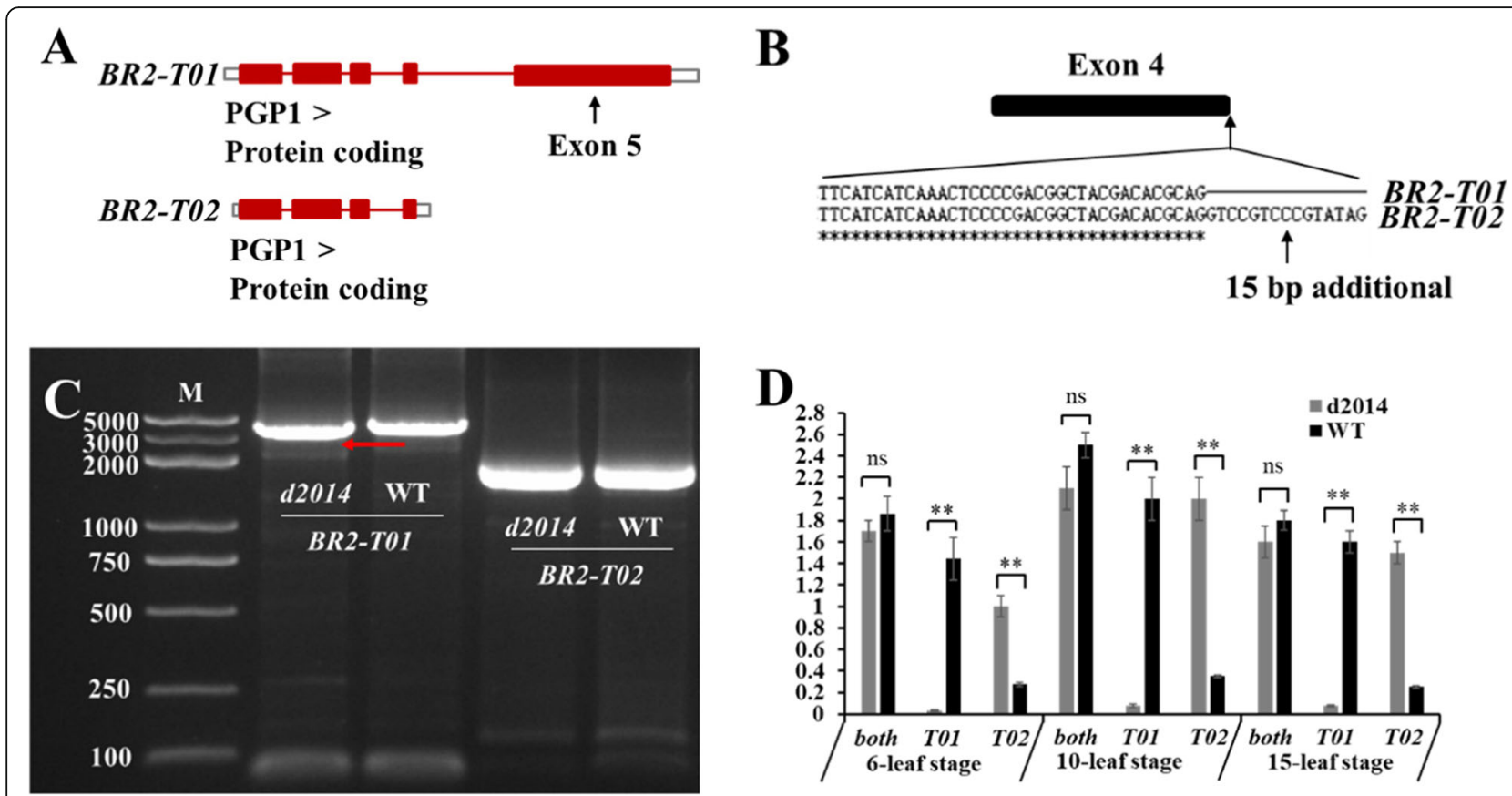

Fig. 3 Identification of the two transcripts of BR2. a Distribution of the exons of BR2-T01 and BR2-T02. $\mathbf{b}$ Differences in exon4 between BR2-T01 and BR2-T02. c Molecular identification of BR2-T01 and BR2-T02. The red arrow indicates the differences of the band size between mutant d2014 and WT for BR2-T01. d The expression pattern of BR2-T01 and BR2-T02 in d2014 and WT. ${ }^{* *} P \leq 0.01$ (Student's t-test); ns, not significant

seriously inhibited. At the 6-leaf stage, the first overground internode (the 6th internode) was elongating. Though it seemed to be very short (Fig. 6a), there was a trending difference between WT and $d 2014$. In order to understand the cell morphology of these shortened internodes in $d 2014$, we observed the young 6th internode at the 6-leaf stage. Cross-sections showed that $d 2014$ had regular parenchyma cells similar to WT, and their cell sizes were comparable, whereas the numbers of vascular bundles in $d 2014$ had been significantly reduced (Fig. 7a, c). Longitudinal sections showed that the length of parenchyma cells was not changed between $d 2014$ and WT, but the interval of two adjacent vascular bundles in $d 2014$ was larger than that in WT (Fig. 7b), which meant that there was a reduced vascular bundle number for $d 2014$. Thus, the $d 2014$ mutation originally affects the formation of vascular bundle in shortened internode cells.

\section{Location of $B R 2$ expression in the stem}

Since the mutant $d 2014 / b r 2$ displays defects in internode elongation, we predicted that $B R 2$ is expressed in these tissues. However, the expression pattern of $B R 2$ has not been precisely defined previously. To figure out the location of $B R 2$ expression in the stem, we performed in situ RNA hybridization at the 10-leaf stage. In longitudinal sections of the stem apex, $B R 2$ expression was clearly localized in each node (Fig. 8a). The upper and lower nodes revealed clear signals in the single internode on the ear (Fig. 8b). In cross sections of the longest internode, BR2 mRNAs were observed in a mass of vascular bundles (Fig. 8c). A single internode was selected for control experiments, in which the sense probe produced no signal (Fig. 8d, e). In maize stems, the vascular bundles interlace in nodes and then tilt into internodes with multiple branches, which finally form a network pipe from top to bottom. Thus, BR2 expression was mainly localized in vascular bundles of the node and internode. Xing indicated that the subcellular localization of QPH1 (BR2) is exclusively in membrane [33]. There is no variation in the signal peptide region of $d 2014 / b r 2$, thus, mutation should not influence the subcellular localization of $d 2014 / b r 2$. In WT, the expression level, localization and transport activity of BR2 are normal, relatively; while under other circumstances (for example $b r 2$ mutation), developmental defects occur. Overall, these data indicated that PGP1 functions in auxin export from vascular bundles in stem, then regulating internode elongation.

\section{Quantitative analysis of auxin in the $d 2014$ stem}

Auxin transport influences the distribution and local concentration of auxin [38]. In the prior studies, $b r 2$ mutation influences auxin transport and local concentration, which are responsible for the dwarf phenotype [32-34]. The defective auxin transport in $d 2014$ stems, mediated by BR2/PGP1, would cause the auxin concentration variation in the stem and then influence the 
A

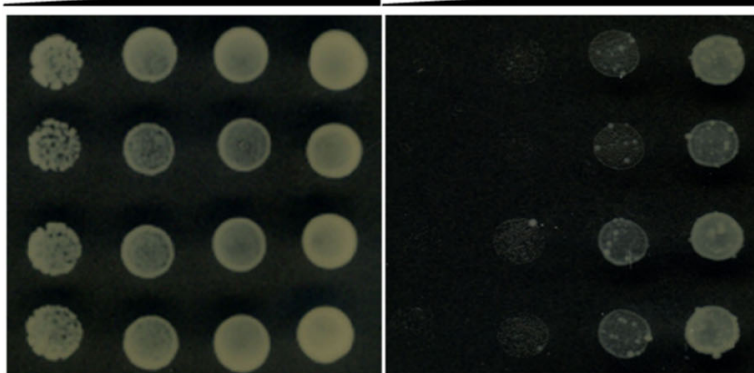

gef1

vector

pYHE-d2014-PGP1-T01

pYHE-WT-PGP1-T01

5-FI: $0 \mu \mathrm{M}$

5-FI: $250 \mu \mathrm{M}$

B

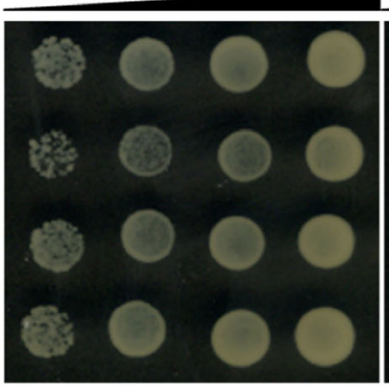

5-FI: $0 \mu \mathrm{M}$

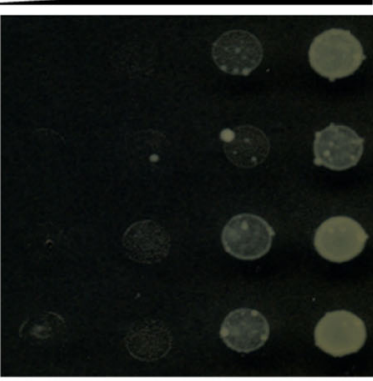

5-FI: $250 \mu M$ gef1

vector

pYHE-d2014-PGP1-T02

pYHE-WT-PGP1-T02

Fig. 4 Functional analysis of protein PGP1-T01 and PGP1-T02. $\mathbf{a}$, b Heterologous expression of PGP1-T01 and PGP1-T02 for detoxification assays in 5-Fl hypersensitive gef1 mutant strain, respectively. Represents that 1-, 10-, 100-, and 1000-fold dilutions (from right to left) of yeast cells were orderly spotted on YPDA medium containing $0 \mu \mathrm{M}$ or $250 \mu \mathrm{M} 5$-Fl, including the original strain gef1, the vector control and the transformed strains

internode elongation. To investigate the links between the two, we detected the auxin concentration in several key periods of internode elongation variation (as mentioned above). At the 6-leaf stage, auxin concentration in the $d 2014$ stem apex was significantly higher than that in WT (Fig. 9a). We also measured the auxin concentration in the stem apex of $d 2014$ and WT at the 12-leaf and 14-leaf stages. However, auxin concentration was gradually reduced compared with the former period (Fig. 9a). Moreover, little difference was observed between $d 2014$ and WT at these two stages (Fig. 9a). These showed that the $d 2014 / b r 2$ caused higher auxin level in the stem apex at the early development stages, at least at the 6-leaf stage, and correspondingly, the internode elongation of $d 2014$ was suppressed. In addition, we examined the auxin levels of the single ear-internode at the 14-leaf stage, including two parts: the node and the internode region. Interestingly, the auxin level of the nodes in $d 2014$ was significantly higher than the nodes in WT, as well as the internode region itself (Fig. 9b), while in WT, there was no significant difference (Fig. 9b). Furthermore, the auxin levels of the two parts exceeded the stem apex at the 14-leaf stage (Fig. 9a; b).
These data revealed that auxin at the 14-leaf stage was increased for ear-internode and was detained in the node region of $d 2014$. Notably, the ear-internode in $d 2014$ began to grow rather slowly after the 14-leaf stage and finally was extremely shortened. Taken together, these data showed that the $d 2014 / b r 2$ caused increasing in auxin levels in the shortened lower internodes (stem apex at the 6-leaf stage) and ear internodes (node region at the 14-leaf stage), which were responsible for the defects of internode elongation.

\section{Discussion}

d2014 gene, with a splicing variation, is a potentially useful br2 allele

In maize, there have been many reports on the identification of $b r 2$ mutants, which involved a variety of the mutation loci (Additional file 5: Figure S3). At first, the $B R 2$ gene were cloned and confirmed by transposon tagging with Mutator $(M u)$, such as br2-6, br2-7 and br29 with $M u$ insertion in exon 1 , as well as $b r 2-3$ with $M u$ insertion in intron 4 [32]. Subsequently, several other br2 alleles, such as one SNP variant (G/T5295) in exon 5 [33] and a $241 \mathrm{bp}$ deletion (G6367 to C6617) in exon 5 


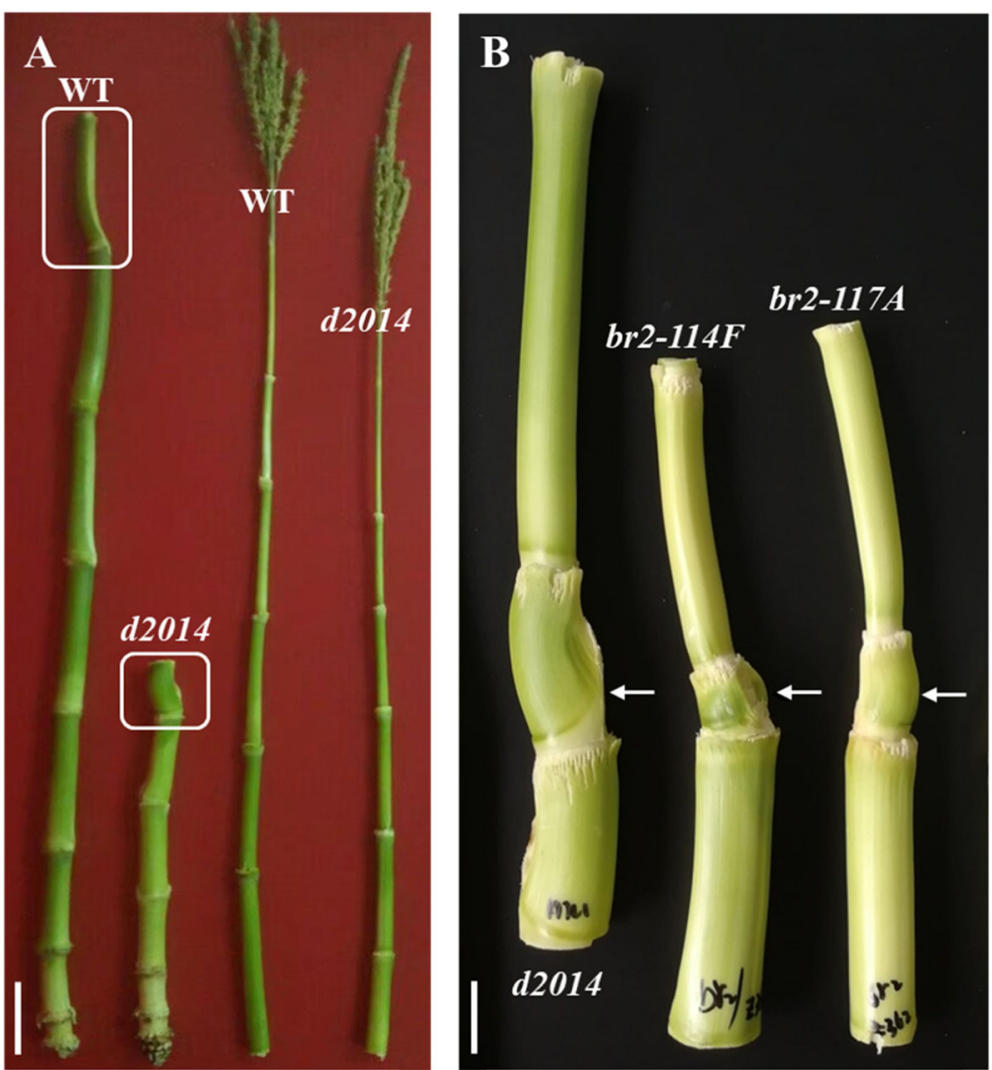

Fig. 5 Morphological comparison of internodes at the mature stage. a Morphology of the internodes below the ear or above the ear between d2014 and WT. The white box indicates ear-internode. Bar $=10 \mathrm{~cm}$. b Morphology of the ear-internodes of the three br2 mutants: d2014, br2-114F, and br2-117A. The arrows indicate the ear-internodes. Bar $=2 \mathrm{~cm}$

[39], were identified to result in the partial loss of BR2/ PGP1 protein function. All the above $b r 2$ mutants were derived from multiple inbred lines with a different genetic background. Nevertheless, they all presented shorter lower internodes, but nearly normal upper internodes. Without exception, our $d 2014 b r 2$ displayed similar features, which were verified by allelism test with $b r 2-114 F$. Apart from the above phenotypes, we also found that the $b r 2$ mutants $(d 2014, b r 2-114 F$ and br2-117A) displayed a shortened ear-internode. Therefore, it is certain that this $b r 2$ allele is responsible for the shortened internodes.

In the maize $B 73$ reference genome, $B R 2$ has two predicted splicing variants: $B R 2-T 01$ and BR2-T02. In this study, we identified a new $b r 2$ allele (d2014), which contained a $546 \mathrm{bp}$ insertion in $B R 2$ intron $3\left(\mathrm{C}_{2073}\right)$ and a 233 bp deletion $\left(\mathrm{A}_{6568}\right.$ to $\left.\mathrm{C}_{6800}\right)$ in $B R 2$ exon 5 . Sequencing of the cDNA confirmed the presence of the two $B R 2$ splicing variants, and $B R 2-T 02$ was highly expressed in $d 2014$ while BR2-T01 expression level was very low, which was contrary to that in WT. Similarly, the two BR2 splicing variants have been verified molecularly in a dwarf br2-NC238 allele mutant [40]. The insertion of a novel transposon in $B R 2$ intron 4 alters the normal splicing of the gene, which results in abundant expression of $B R 2$ T02 in $b r 2-N C 238$ instead of BR2-T01 in tall NC238 plants. Generally, insertion, deletion, or single-base replacement of the exon region may result in the defective protein with altered function; while the variation of the intron region might affect gene expression pattern through changing the mRNA splicing. Therefore, we concluded that the insertion of $546 \mathrm{bp}$ in intron 3 altered the normal splicing of $B R 2$ gene, which was the main reason for the dwarfing of $d 2014$. Overall, $d 2014$ with a splicing variants has the enriched types of $b r 2$ allele.

Some studies have shown that some different splicing variants perform equivalent functions to the constitutive form, even when lacking amino acid sequences or protein domains [41]. The BR2 gene encodes an auxin transport protein, PGP1, which is an ATP-binding cassette (ABC) transporter [42]. In general, full-size $\mathrm{ABC}$ transporters consist of two similar halves, each containing a transmembrane domain (TMD) and a nucleotide binding domain (NBD) [43]. The so-called half-size ABC transporters only have a half (one TMD and one NBD) and require dimerization for their transport activity [43]. An example 
A

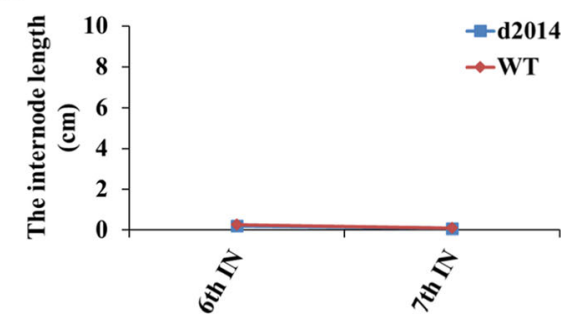

C

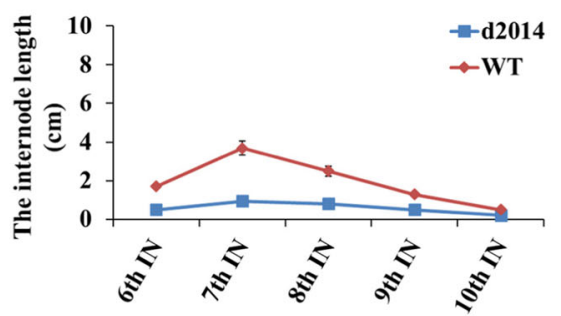

E

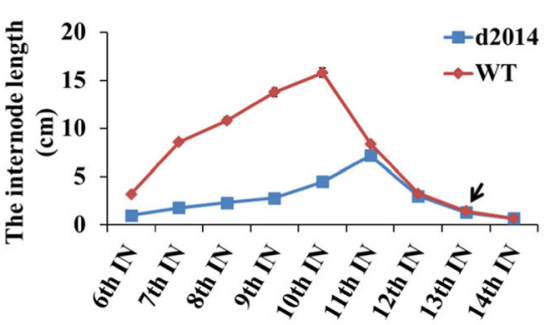

G

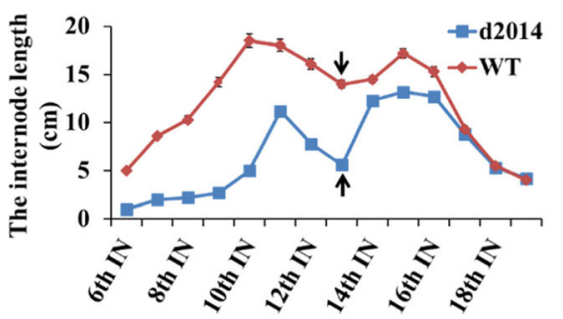

B

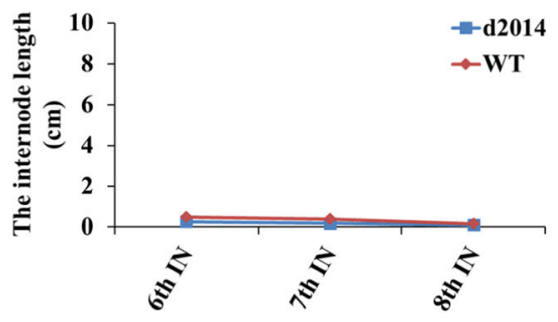

D

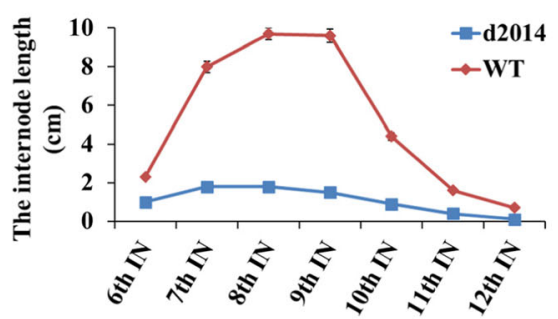

F

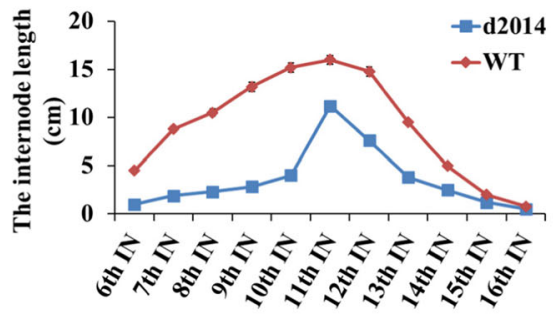

H

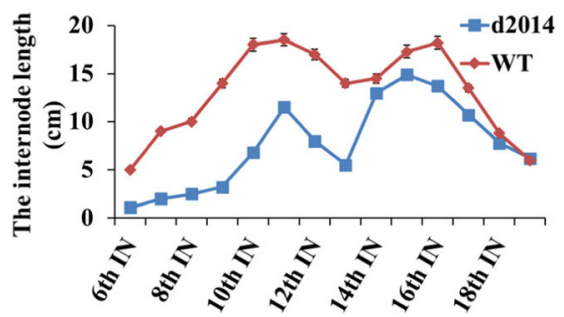

Fig. 6 Dynamic observation of internode elongation. $\mathbf{a}, \mathbf{b}, \mathbf{c}, \mathbf{d}, \mathbf{e}, \mathbf{f}, \mathbf{g}, \mathbf{h}$ The length of each internode at the 6-, 8-, 10-, 12-, 14-, 16-, 18-, 20-leaf stages, respectively. IN represents internode. At the 6-leaf stage, the first internode (6th internode) above ground is visible and then more internodes are elongated subsequently at other stages. The arrows in $\mathbf{e}$ and $\mathbf{g}$ indicate the ear-internode (13th internode)

is ECERIFERUM5, which is a half-size ABC protein and functions in wax export to the plant cuticle [44]. Here, through the analysis of protein domains by (http://smart. embl-heidelberg.de/smart), we found that PGP1-T01 was a full-size ABC transporter, while PGP1-T02 contained exactly a half, making it a half-size ABC transporter (Additional file 6: Figure S4). Diana Santelia and Markus Geisler examined the auxin transport properties (influx or efflux) of $\mathrm{ABCB}$ protein by heterogeneous expression in yeast $[35,36]$. Since the strong promoter was used to induce the overexpression of $\mathrm{ABCB}$ protein, it would not accurately evaluate the auxin transport activity. Similarly, our heterogeneous expression assays using strong promoter identified the transport properties of the two proteins PGP1-T01/T02, which indicated that PGP1-T01 (even including the defective d2014-PGP1-T01) and PGP1-T02 have played similar roles in auxin efflux. Previously, Multani and Knöller have confirmed that br2 mutation leads to the decrease of auxin flow from top to bottom, which is responsible for the dwarf phenotype [32, 34]. In our study, the expression of br2-T01 in $d 2014$ was significantly lower than that of BR2-T01 in WT (normal 


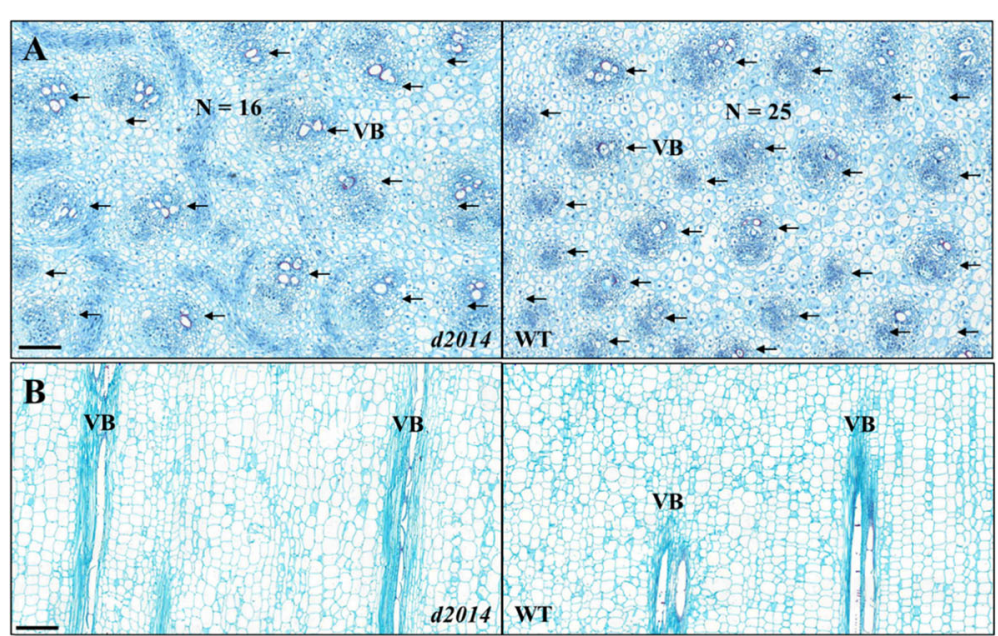

C

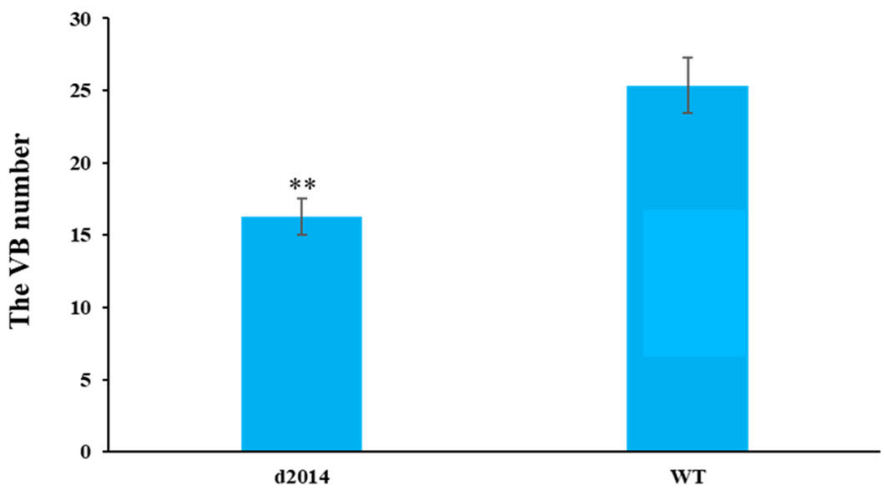

Fig. 7 Morphological observation of the 6th internode cells of d2014 and WT at the 6-leaf stage. a Cross-section of cells of d2014 and WT. The arrows point to the vascular bundle (VB); N represents the total VB numbers. The magnification times are $20 \times$. Bar $=150 \mu \mathrm{m}$. $\mathbf{b}$ Longitudinal section of cells of $d 2014$ and WT. The magnification times are $20 \times$. Bar $=200 \mu \mathrm{m}$. c The statistics of VB number for the fixed center cross section. ** indicates the VB numbers in $d 2014$ were significantly reduced at $P \leq 0.01$ level

status), indicating that the normal auxin transport activity in $d 2014$ was impaired. Nevertheless, our phenotypic analysis showed that $d 2014$ was a mild dwarf mutant with nearly no other undesirable traits, which should be attributable to the compensation of functional PGP1-T02. Actually, in the $\mathrm{F}_{2}$ population of $b r 2-q p h 1$ and $b r 2-117 A$, plant height and ear height segregated in a 3:1 ratio $(b r 2-$ qph1/- plants to $b r 2-117 A / b r 2-117 A$ plants), which indicated that br2-qph1 was dominant to $b r 2-117 A$ [33]. Namely, different $b r 2$ alleles would give rise to certain defects in varying degrees. Therefore, our work shows a useful $b r 2$ allele that has beneficial potential for maize improvement by moderately reducing the plant height while not affecting the yield.

\section{BR2/PGP1 functions in auxin efflux from the vascular bundles of the node and internode}

Auxin is often synthesized in the apex region and is directionally transported to the lower region $[45,46]$. This directional auxin flow is attributable to membranous auxin transporters. So far, three major families of auxin transporters have been identified: AUXIN-RESISTANT1 (AUX1)/AUX1-LIKEs (LAXs) for auxin influx and PINFORMEDs (PINs) and several PGP proteins for auxin efflux [47]. Some studies indicated that $A B C B$ efflux transporters might limit auxin reuptake at efflux sites for assisting PIN-oriented auxin flow [48]. In addition, several studies suggested that ABCBs may play a role in local auxin loading of long-distance auxin transport $[49,50]$.

BR2/PGP1 is an orthologue of Arabidopsis thaliana ABCB1/PGP1, which is expressed in shoot and root apices and functions primarily in auxin efflux from meristematic cells into long-range auxin transport streams $[34,51,52]$. Previously, Knöller applied $\left[{ }^{3} \mathrm{H}\right]$-IAA to the upper stem of maize (B73 vs $b r 2$ ), and found that several $\left[{ }^{3} \mathrm{H}\right]$-IAA accumulation peaks were detected near the nodes; meanwhile, the moving front was reduced and 


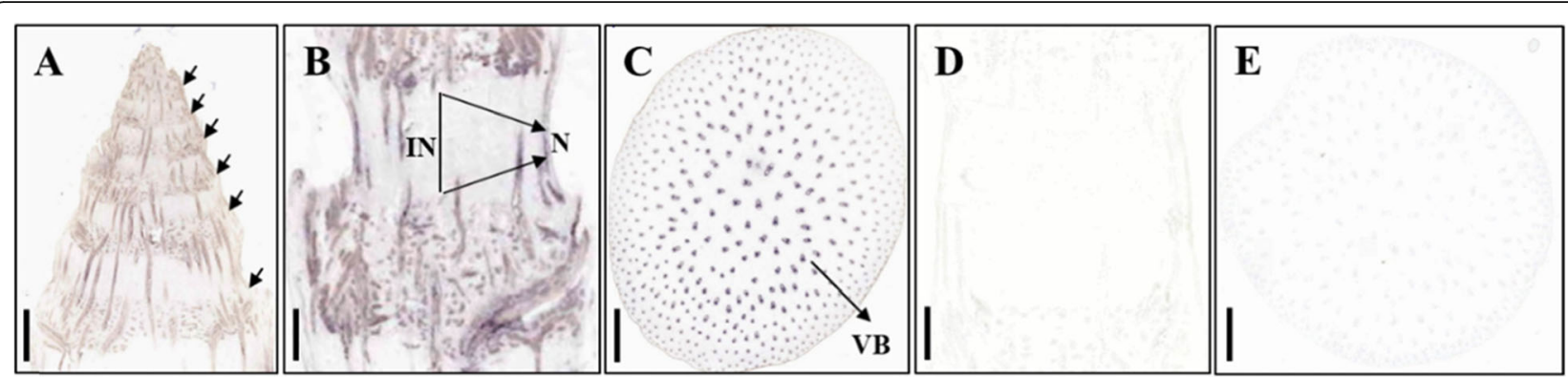

Fig. 8 In situ localization of BR2 mRNA in stem at the 10-leaf stage. a Longitudinal section of stem apex. The arrows represent each node in stem apex. Bar $=0.1 \mathrm{~cm}$. b Longitudinal section of an intact internode. IN, internode; N, node. Bar $=0.3 \mathrm{~cm}$. c Transverse section of longest internode. VB, vascular bundle. $B a r=0.2 \mathrm{~cm}$. d, e Hybridization of a single internode with a $B R 2$ sense probe for longitudinal section and transverse section, respectively. Bar $=0.2 \mathrm{~cm}$

free auxin levels in the node of $b r 2$ mutant are significantly higher than in adjacent internodes [34]. Consistently, in our $d 2014 / b r 2$, higher auxin levels were detected in the stem apex (including multiple nodes) at the 6-leaf stage and in the node region of the earinternode at the 14-leaf stage. In a word, the $b r 2$ mutation caused auxin accumulation in the node regions. Notably, our in situ hybridization assay showed that $B R 2$ is primarily expressed in vascular bundles (VBs) of the nodes and internodes. In line with this, Anne Sophie Knoller indicated that BR2 is mainly expressed in the nodal region (dense VBs) [34]. In this context, we propose that BR2/PGP1 functions in auxin efflux from the node to the internode through vascular bundles. Thus, auxin efflux is reduced from the nodes to the internodes in $b r 2$ mutant, and auxin level is higher in $b r 2$ node regions.

Excessive auxin levels in the intercalary meristem region suppress internode elongation

Internode elongation is controlled by several factors, mainly the plant hormones. In this study, we found that the variation of auxin level in $d 2014$ stem influences internode elongation. At the 6-leaf stage, the elongation of the lower internodes began to be severely suppressed in $d 2014$, along with higher auxin levels in the stem apex compared with WT. As the auxin levels of the stem apex gradually decreased at the 12-leaf and 14-leaf stages, internodes in $d 2014$ grew rapidly. At the 14-leaf stage, additionally, the auxin level of the node region next to the ear in $d 2014$ was much more than that in WT, corresponding to the irregular and shortened earinternode. In short, $d 2014 / b r 2$ and WT have the same genetic background except the $b r 2$ locus, causing the shortened internodes, as well as higher auxin levels in their node regions, which suggested that higher auxin levels in $d 2014$ stems (node regions) suppress the internode elongation.

Generally, auxin has fundamental roles in rapid stimulation of cell expansion for promoting growth [53, 54]. This seems to be contradictory with what we found in this study. Nevertheless, similar scene that auxin inhibits growth is fit for apical dominance in plant. Growing shoot apexes produce an inhibitory hormone, auxin, which moves downwards within the stem and inhibits the growth of axillary buds (including the AM) next to

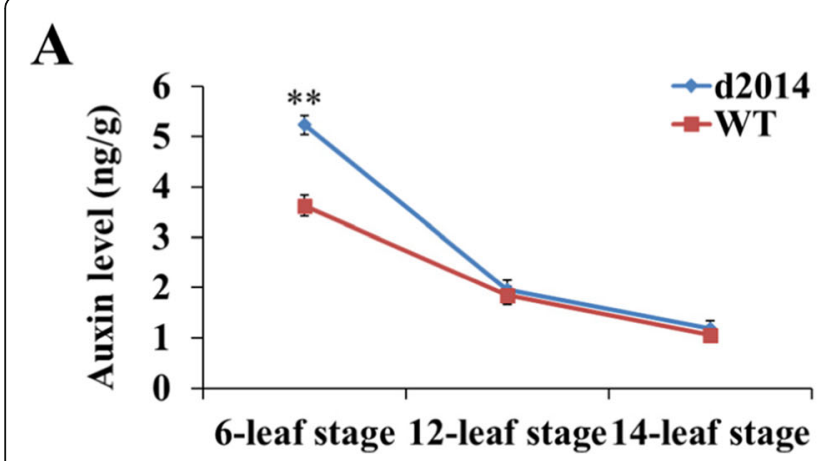

B

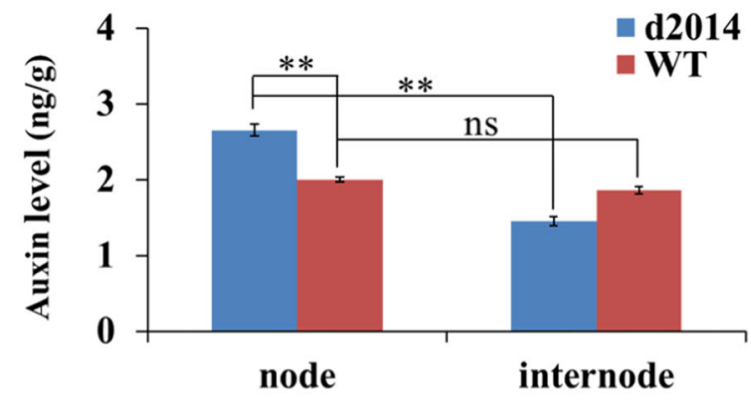

Fig. 9 The change of auxin concentrations in the stem at different stages. a Auxin levels of stem apex in d2014 and WT at the 6-, 12-, and 14-leaf stages. b Auxin levels of a single ear-internode including node and internode region at the 14-leaf stage. ** indicates significant difference at 0.01 level by Student's t-test. ns, not significant 
the node $[55,56]$. Recent studies have revealed that a local auxin minimum is critical for AM formation or initiation [57-59]. Correspondingly, the stem of some plants, including maize, contains intercalary meristems that support stem growth independently of the shoot apex $[5,60]$. Intercalary meristems are located in the internodes immediately above a node, by which the internode can elongate [61]. Moreover, our cytological analysis showed that the $d 2014$ mutation causes a reduced number of vascular bundles, which should be related to intercalary meristem functions. In concordance with this, Multani has also observed the cellular architecture of the $b r 2$ internodes, and their data showed that the VB numbers of 1th over-ground internode (6-weekold) are reduced [32]; at the same time, Xing found that the VB numbers of 6th over-ground internode (19-leaf stage) are altered (reduced without specific statistics) in br2 mutants [33], which indicated that less-elongated internodes in $b r 2$ have reduced VB numbers. Some studies have shown that cell division or differentiation dependent on auxin transport flow is crucial for the formation of vascular bundles in the leaf [62]. Therefore, we speculate that a low auxin level is indispensable for intercalary meristem functions and, in reverse, internode elongation is suppressed when excessive auxin is arrested in intercalary meristem region.

All the $b r 2$ mutants presented shortened lower internodes, as well as ear-internode, but nearly normal upper internodes. Through dynamic observation of internode elongation, we found that the variation of internode elongation in $d 2014$ is closely related to the 6-leaf, 12 leaf and 14-leaf stages. Notably, at the 6-leaf stage, the first internode (6th internode) above the ground was visible, and more internodes elongated subsequently (Additional file 7: Figure S5A); whereas, the juvenile tassel came into view at the 12-leaf stage (Additional file 7:
Figure S5B); in addition, the ear-internode, at the 14-leaf stage, was undetectable until the upmost AM emerged (Additional file 7: Figure S5C). These three stages involve the growth and development of shoot apical meristem (SAM), inflorescence meristem (IM) and AM, which is associated with the synthesis of auxin. In context, we present a model to illuminate how loss-offunction of $b r 2$ alleles uniquely regulates internode elongation (Fig. 10). At early stages, vigorous auxin is synthesized in the SAM region, and then excessive auxin is arrested in the intercalary meristem region of the lower internodes, and finally, elongation of the lower internodes is suppressed. Along with SAM translating to $\mathrm{IM}$, declining auxin is generated in the apex region, and the upper internodes grow normally. For the severely shortened ear-internode, the chief culprit is a new auxin source derived from the developmental AM, which promotes abundant auxin being restricted in the intercalary meristem region of the ear-internode. Overall, the BR2/ PGP1-dependent low auxin level in the intercalary meristem region is crucial for internode elongation.

\section{Conclusions}

Plant height is one of most important agronomic traits in crop breeding. Maize BR2/PGP1 regulates the plant height by promoting auxin efflux from node to internode and avoiding excessive auxin accumulation in the intercalary meristem region to suppress the internode elongation. These findings are of great significance to the decryption of genetic mechanism of plant height and the improvement of maize yield.

\section{Methods}

Plant materials

The $d 2014$ is a dwarf mutant derived from the HL9047 inbred line (as WT), which was bred by our pedigree

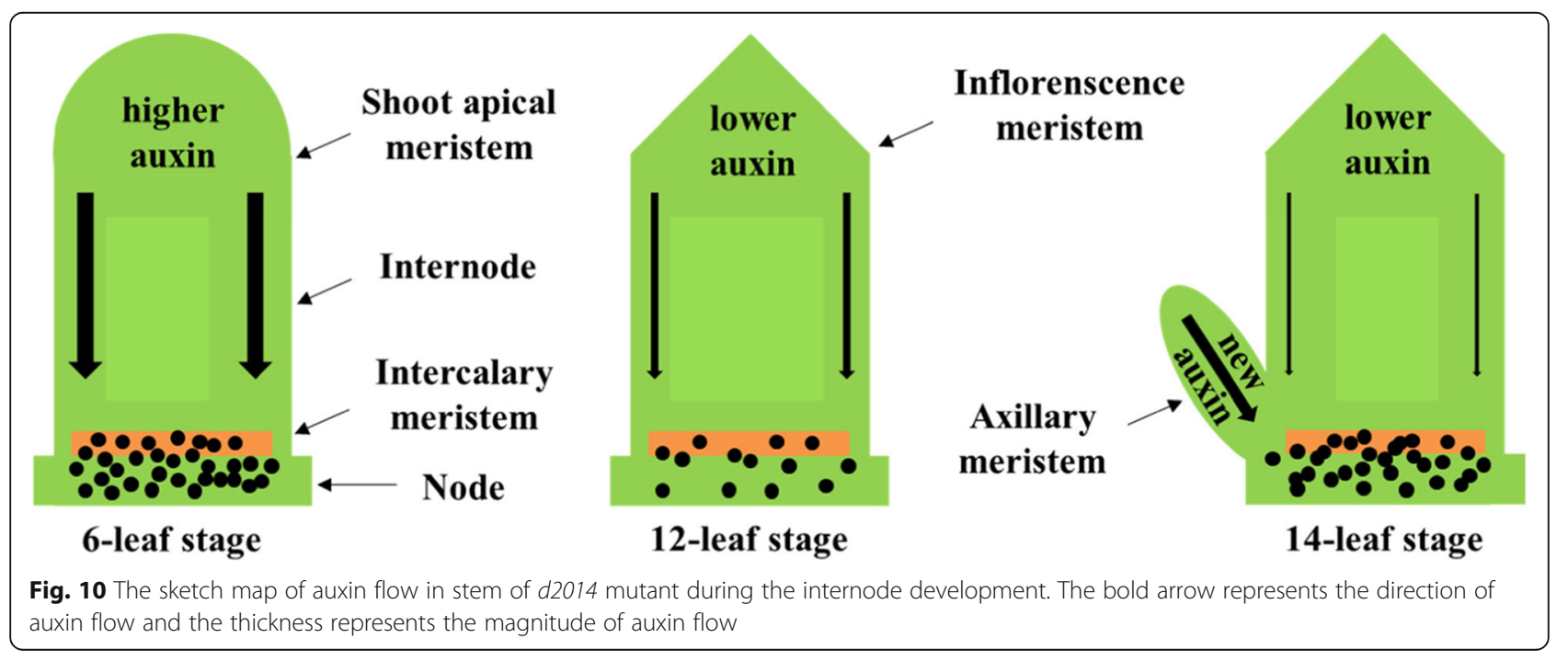


method after 9-generation selection. Three different tall stature inbred lines (HL5038, HL5054 and F19) were selected from our high generation breeding materials to construct $\mathrm{F}_{2}$ and $\mathrm{BC}_{1}$ populations with d2014. In addition, the br2 mutants were requested from the Maize Genetic Cooperation Stock Center (stock number: $114 \mathrm{~F}$ and $117 \mathrm{~A}$ ), the $b r 2-114 F$ and $b r 2-117 A$ were identified and amplified by our group for $b r 2$ allelism test. All the plants were permissively cultivated in the standard experimental field of Sichuan Agricultural University in Sichuan during the summer and in Yunnan during the winter.

\section{Positional cloning of the $d 2014$ gene}

The $\mathrm{BC}_{1}$ population $(\mathrm{HL} 5054 \times d 2014) \times d 2014$ was used, and 6000 individuals were prepared to define the $d 2014$ gene locus. Indeed, using the simple sequence repeat (SSR) makers obtained from the MaizeGDB Database and Indel molecular makers acquired from Liu Jian research group in Sichuan Agricultural University, d2014 gene was mapped to chromosome 1 bin 1.06, a $510 \mathrm{~Kb}$ region with $B R 2$ and six other predicted genes. To identify the mutation site(s) of $d 2014$ gene, we amplified the DNA sequences of these genes and compared them between $d 2014$ and WT. Meanwhile, we used the $b r 2$ mutant $114 \mathrm{~F}$ to examine the allelism between $d 2014$ gene and $B R 2$.

\section{RNA isolation and quantitative real-time PCR (qRT-PCR) analysis}

At the 6-leaf, 10-leaf and 15-leaf stages, stem apexes (about $0.5 \mathrm{~cm}$ ) in $d 2014$ and WT were flash-frozen by liquid nitrogen and stored at $-70{ }^{\circ} \mathrm{C}$. Thereinto, the stem apexes are the top internodes except the juvenile tassel at the 10-leaf and 15-leaf stages. Total RNA was extracted using Trizol reagent, and the quantity and purity of RNA was determined by a NanoDrop 2000 and 1\% agarose gel electrophoresis. Then, RNA was reverse-transcribed to cDNA using the PrimeScript ${ }^{\mathrm{m}}$ RT-PCR Kit with gDNA Eraser (TaKaRa). The database showed that $B R 2$ has two splicing forms corresponding to two transcripts: BR2-T01 and BR2-T02 (https://www.maizegdb.org/). PCR amplification of these CDSs was performed, and the PCR products were detected by electrophoresis and then sequenced. In addition, qRT-PCR was performed in a Bio-Rad CFX96 Real-Time PCR System with SYBR Green PCR Master Mix (TaKaRa). Specific primers were designed for distinguishing BR2-T01 and BR2-T02. The primer Q-BR2-T01 was designed to span exons 4 and 5 , while the primer QBR2-T02 covered exon 3, exon 4, and the 3'UTR region of $B R 2-T 02$. In addition, we designed the primer Q-BR2both, which covered exons 3 and 4, for detecting the total $B R 2$ expression level. After normalization to ACTIN1 expression as an internal control, the relative expression levels were measured through the $2^{-\Delta \Delta C t}$ method. All primer sequences are listed in Additional file 1: Table S1.

\section{Heterogeneous expression assays in yeast}

The yeast mutant strain gefl, which is hypersensitive to the IAA toxic analog 5-Fluoroindole (5-FI), was used to explore auxin transport properties [35, 36]. Yeast gef1, ATCC $^{\circ} 4036838^{\text {tm }}$, was ordered from the American Type Culture Collection (ATCC). Firstly, yeast shuttle vector pGADT7, which has two HindIII restriction enzyme cutting sites, was digested by HindIII to remove the nuclear localization signal (NLS) sequence. In addition, the expression of the transcript on vector pGADT7 is driven by a strong promoter $\mathrm{P}_{A D H 1}$, so the yeast transgenics are similar to the overexpressed lines. Then, the CDSs of BR2-T01 and BR2-T02 were acquired and inserted into pGADT7-HindIII by homologous recombination (singleor multi-fragment), generating pYHE- $d 2014-\mathrm{PGP1}-\mathrm{T} 01$, pYHE-d2014-PGP1-T02, pYHE-WT-PGP1-T01 and pYHE-WT-PGP1-T02. All the recombinant vectors were introduced into the yeast mutant strain gef1. For leucine-deficient type gef1, single colonies were screened in synthetic minimal medium without leucine (SD$\mathrm{Leu}^{-}$). Lastly, transformants grown in SD-Leu to $\mathrm{OD}_{600}=0.8$ were washed and resuspended in water to $\mathrm{OD}_{600}=1.0$. Cells were 10 -fold, 100 -fold, 1000 -fold diluted, and $2.5 \mu \mathrm{l}$ of each was spotted on YPDA medium containing $0 \mu \mathrm{M}$ or $250 \mu \mathrm{M} 5$-FI [36] with the corresponding vector control and the yeast mutant strain itself as controls. Pictures were taken after 3-6 days of growth at $28^{\circ} \mathrm{C}$. All primer sequences in homologous recombinant are listed in Additional file 1: Table S1.

\section{Dynamic observation of internode elongation}

Each $700 d 2014$ and WT individuals were planted in a specially designed plot. The plot consisted of 3-m-long rows separated by $1 \mathrm{~m}$ space between each row. Fiverow interval planting was performed for $d 2014$ and WT with 10 replicates, and 14 individuals were planted in each row. The 5th leaf and 10th leaf of uniform individuals were labelled in the field. In general, the first internode above the ground is visible when the 6th leaf is unfolded at the 6-leaf stage. Thus, we measured each visible internode length from the 6-leaf stage to the maturation stage, including the $6,8,10,12,14,16,18$, and 20-leaf stages. At each stage, 10 leaf-labelled individuals in adjacent rows were investigated, and the average value was the phenotypic value of internode corresponding to the leaf number.

\section{Histocytological analysis}

A plenty of WT and mutant individuals were planted (as described above). At the 6-leaf stage, the first overground internode (the 6th internode) elongated. We 
randomly picked three individuals of WT and mutant, respectively, as three biological replicates for cytological observation of the internodes. The whole 6th internode was cut except for two terminals (the node regions) to make a paraffin section. All samples were vacuumed quickly and fixed in FAA composed of 5\% of formaldehyde $(40 \% \mathrm{v} / \mathrm{v}), 5 \%$ of acetate, and $90 \%$ of $75 \%$ alcohol $(\mathrm{v} / \mathrm{v})$ overnight. The paraffin sections were made as described previously [67]. Firstly, the transection was made from the middle part (about $0.1 \mathrm{~cm}$ from the upper part). Next, the longitudinal section was made from the rest of the upper part. The cell morphology was observed through an optical microscopy. Based on the same area (the middle region) and the same magnification $(20 \times)$, we counted all the vascular bundles (VBs) for the transection.

\section{In situ hybridization analysis}

At the 10-leaf stage, tissues of WT were prepared, including the stem apex (about $0.5 \mathrm{~cm}$ segment from the tip), longest internode and single internode (about 1 $\mathrm{cm})$. In situ hybridization for $B R 2$ was performed as described previously [63]. An antisense RNA probe labeled by the Digoxin marker was uniquely designed in the $B R 2$ exon 1 region, and sequences are listed in Additional file 1: Table S1. To reduce the disturbance of the background signal, pilot experiment was conducted to optimize the probe dosage. In addition, a single internode was selected for the control test with a sense probe (Additional file 1: Table S1).

\section{Endogenous auxin concentration analysis}

The stem apex segment (about $0.5 \mathrm{~cm}$ ) was prepared in several periods, including 6-, 12-, and 14-leaf stages. All tissues were flash-frozen by liquid nitrogen and stored at $-70^{\circ} \mathrm{C}$. The measurements of endogenous auxin were performed through liquid chromatography tandem mass spectrometry (LC-MS). Three repeats with three plants in each replicate were performed. Furthermore, at the 14-leaf stage, the ear-internode was divided into two parts, including the basal node segment (about $0.2 \mathrm{~cm}$ ) and the middle internode segment (about $0.2 \mathrm{~cm}$ ), the auxin concentration of which was also measured as above.

\section{Supplementary information}

Supplementary information accompanies this paper at https://doi.org/10. 1186/s12870-019-2200-5.

Additional file 1: Table S1. Probe sequences for $B R 2$ in situ hybridization and primers used for sequencing and GRT-PCR as well as homologous recombinant.

Additional file 2: Table S2. Plant height separation performance in $F_{2}$ and $\mathrm{BC}_{1}$ populations.
Additional file 3: Figure S1. The $b r 2$ allelism test for $d 2014$

Additional file 4: Figure S2. Comparison of certain internode length between d2014 and WT at the 12-leaf, 14-leaf, and 20-leaf stages.

Additional file 5: Figure S3. Different mutation sites of br2 alleles. Additional file 6: Figure S4. The analysis of protein domains of PGP1T01 and PGP1-T02.

Additional file 7: Figure S5. The morphological characteristics of the stems of d2014 and WT.

\section{Abbreviations}

5-Fl: 5-Fluoroindole; ABC: ATP-binding cassette; AMs: Axillary meristems; an1: Anther ear1; ATCC: American Type Culture Collection; AUX1: AUXINRESISTANT1; br2: Brachytic2; BRs: Brassinosteroids; d1: Dwarf1;

d2014: Dwarf2014; dw3: Dwarf3; GAs: Gibberellins; IM: Inflorescence meristem; LAXs: AUX1-LIKEs; LC-MS: Liquid chromatography tandem mass spectrometry; Mu: Mutator; naz: Nana plant2; NBD: Nucleotide binding domain; NLS: Nuclear localization signal; PGP1: P-glycoprotein1; PINs: PINFORMEDs; qRT-PCR: Quantitative real-time PCR; SAM: Shoot apical meristem; SLs: Strigolactones; SSR: Simple sequence repeat;

TMD: Transmembrane domain; VBs: Vascular bundles; vt2: Vanishing tassel2; WT: Wild type

\section{Acknowledgements}

This work was financially supported in part by National Key Laboratory of Wheat and Maize Crop Science in Henan Agricultural University and the National Natural Science Foundation of China (No.31571684). We thank Chengdu Diversity Co. Lit (Chengdu Sichuan) for the measurement of the endogenous hormone auxin.

\section{Authors' contributions}

YBH and JHT designed the research. XGZ, XBH, YHL, LJZ, QY, HJZ, XRH, FLZ, and LC performed the experiments. XGZ and XBH wrote the manuscript, JJZ, YFH, HML, YPL, GWY and HHH revised the manuscript. All authors read and approved the final manuscript.

\section{Funding}

This work was financially supported in part by National Key Laboratory of Wheat and Maize Crop Science in Henan Agricultural University and the National Natural Science Foundation of China (No.31571684). The funding body was involved in the material creation and designing the study.

\section{Availability of data and materials}

All data generated or analyzed during this study are included in this published article and its additional files.

Ethics approval and consent to participate Not applicable.

\section{Consent for publication}

Not applicable.

\section{Competing interests}

The authors declare that they have no competing interests.

\section{Author details}

${ }^{1}$ State Key Laboratory of Crop Genetics of Disease Resistance and Disease Control, Sichuan Agricultural University, Chengdu 611130, Sichuan, China. ${ }^{2}$ College of Agronomy, Sichuan Agricultural University, Chengdu 611130, Sichuan, China. ${ }^{3}$ College of Agriculture and Food Engineering, Baise University, Baise 533000, Guangxi, China. ${ }^{4}$ Maize Research Institute, Sichuan Agricultural University, Chengdu 611130, Sichuan, China. ${ }^{5}$ College of Life Science, Sichuan Agricultural University, Ya'an 625014, Sichuan, China. ${ }^{6}$ National Key Laboratory of Wheat and Maize Crop Science, Henan Agricultural University, Zhengzhou 450000, Henan, China. 
Received: 8 July 2019 Accepted: 12 December 2019 Published online: 27 December 2019

\section{References}

1. Li H, Wang Y, Li X, Gao Y, Wang Z, Zhao Y, Wang M. A GA-insensitive dwarf mutant of Brassica napus L. correlated with mutation in pyrimidine box in the promoter of GID1. Mol Biol Rep. 2011;38(1):191-7.

2. Salamini F. Hormones and the green revolution. Science. 2003;302(5642):712.

3. Hedden P. The genes of the green revolution. Trends Genet. 2003;19(1):5-9.

4. LV H, Zheng J, Wang T, Fu J, Huai J, Min H, Zhang X, Tian B, Shi Y, Wang G. The maize d2003, a novel allele of VP8, is required for maize internode elongation. Plant Mol Biol. 2014;84(3):243-57.

5. Wang B, Smith S, Li J. Genetic gegulation of shoot architecture. Annu Rev Plant Biol. 2018;69:437-68.

6. Evans MMS, Poethig RS. Gibberellins promote vegetative phase change and reproductive maturity in maize. Plant Physiol. 1995;108(2):475-87.

7. Peng J, Richards D, Hartley N, et al. 'Green revolution' genes encode mutant gibberellin response modulators. Nature. 1999:400(6741):256-61.

8. Lin H, Wang R, Qian Q, Yan M, Meng X, Fu Z, Yan C, Jiang B, Su Z, Li J, Wang $Y$. DWARF27, an iron-containing protein required for the biosynthesis of strigolactones, regulates rice tiller bud outgrowth. Plant Cell. 2009;21(5): 1512-25.

9. Wang Z, Bai M, Oh E, Zhu J. Brassinosteroid signaling network and regulation of photomorphogenesis. Annu Rev Genet. 2012;46:701-24.

10. Guo H, Li L, Aluru M, Aluru S, Yin Y. Mechanisms and networks for brassinosteroid regulated gene expression. Curr Opin Plant Biol. 2013;16(5): 545-53.

11. Bensen R, Johal G, Crane V, Tossberg J, Schnable P, Meeley R, Briggs S. Cloning and characterization of the maize an 1 gene. Plant Cell. 1995;7(1): 75-84.

12. Winkler $\mathrm{R}$, Helentjaris $\mathrm{T}$. The maize Dwarf3 gene encodes a cytochrome P450-mediated early step in gibberellin biosynthesis. Plant Cell. 1995;7(8): 1307-17.

13. Lawit $\mathrm{S}$, Wych $\mathrm{H}, \mathrm{Xu} \mathrm{D}$, Kundu S, Tomes D. Maize DELLA proteins dwarf plant8 and dwarf plant9 as modulators of plant development. Plant Cell Physiol. 2010;51(11):1854-68.

14. Bishop G, Nomura T, Yokota T, Harrison K, Noguchi T, Fujioka S, Takatsuto S, Jones J, Kamiya Y. The tomato DWARF enzyme catalyses C-6 oxidation in brassinosteroid biosynthesis. Proc Natl Acad Sci U S A. 1999;96(4):1761-6.

15. Schultz L, Kerckhoffs L, Klahre U, Yokota T, Reid J. Molecular characterization of the brassinosteroid-deficient Ikb mutant in pea. Plant Mol Biol. 2001;47(4): 491-8.

16. Shimada Y, Fujioka S, Miyauchi N, Kushiro M, Takatsuto S, Nomura T, Yokota T, Kamiya Y, Bishop G, Yoshida S. Brassinosteroid-6-oxidases from Arabidopsis and tomato catalyze multiple C-6 oxidations in brassinosteroid biosynthesis. Plant Physiol. 2001;126(2):770-9.

17. Hong Z, Ueguchi-Tanaka M, Shimizu-Sato S, Inukai Y, Fujioka S, Shimada Y, Takatsuto S, Agetsuma M, Yoshida S, Watanabe Y, Uozu S, Kitano H, Ashikari M, Matsuoka M. Loss-of-function of a rice brassinosteroid biosynthetic enzyme, C-6 oxidase, prevents the organized arrangement and polar elongation of cells in the leaves and stem. Plant J. 2002;32(4):495-508.

18. Best N, Hartwig T, Budka J, Fujioka S, Johal G, Schulz B, Dilkes B. mEncodes a maize ortholog of the Arabidopsis brassinosteroid biosynthesis gene DWARF1, identifying developmental interactions between brassinosteroids and gibberellins. Plant Physiol. 2016;171(4):2633-47.

19. Hu Z, Yan H, Yang J, Yamaguchi S, Maekawa M, Takamure I, Tsutsumi N, Kyozuka J, Nakazono M. Strigolactones negatively regulate mesocotyl elongation in rice during germination and growth in darkness. Plant Cell Physiol. 2010;51(7):1136-42.

20. Benjamins R, Scheres B. Auxin: the looping star in plant development. Annu Rev Plant Biol. 2008;59:443-65.

21. Cheng $Y$, Dai $X$, Zhao Y. Auxin synthesized by the YUCCA flavin monooxygenases is essential for embryogenesis and leaf formation in Arabidopsis. Plant Cell. 2007:19(8):2430-9.

22. Yamada M, Greenham K, Prigge M, Jensen P, Estelle M. The transport inhibitor response2 gene is required for auxin synthesis and diverse aspects of plant development. Plant Physiol. 2009;151(1):168-79.

23. Stepanova A, Robertson-Hoyt J, Yun J, Benavente L, Xie D, Dolezal K Schlereth A, Jürgens $G$, Alonso J. TAA1-mediated auxin biosynthesis is essential for hormone crosstalk and plant development. Cell. 2008;133(1): 177-91.

24. Phillips K, Skirpan A, Liu X, Christensen A, Slewinski T, Hudson C, Barazesh S, Cohen J, Malcomber S, McSteen P. vanishing tassel2 encodes a grass-specific tryptophan aminotransferase required for vegetative and reproductive development in maize. Plant Cell. 2011;23(2):550-66.

25. Kepinski S, Leyser O. Auxin-induced SCFTIR1-aux/IAA interaction involves stable modification of the SCFTIR1 complex. Proc Natl Acad Sci U S A. 2004. 101(33):12381-6.

26. Quint M, Gray W. Auxin signaling. Curr Opin Plant Biol. 2006;9(5):448-53.

27. Hamann T, Benkova E, Bäurle I, Kientz M, Jürgens G. The Arabidopsis bodenlos gene encodes an auxin response protein inhibiting monopterosmediated embryo patterning. Genes Dev. 2002;16(13):1610-5.

28. Hardtke C, Ckurshumova W, Vidaurre D, Singh S, Stamatiou G, Tiwari S, Hagen G, Guilfoyle T, Berleth T. Overlapping and non-redundant functions of the Arabidopsis auxin response factors monopteros and nonphototropic hypocotyl 4. Development. 2004;131(5):1089-100.

29. Dharmasiri N, Dharmasiri S, Estelle M. The F-box protein TIR1 is an auxin receptor. Nature. 2005:435(7041):441-5.

30. Dharmasiri N, Dharmasiri S, Weijers D, Lechner E, Yamada M, Hobbie L, Ehrismann J, Jürgens $\mathrm{G}$, Estelle M. Plant development is regulated by a family of auxin receptor $F$ box proteins. Dev Cell. 2005b;9(1):109-19.

31. Grieneisen $V, X u$ J, Marée A, Hogeweg P, Scheres B. Auxin transport is sufficient to generate a maximum and gradient guiding root growth. Nature. 2007;449(7165):1008-13.

32. Multani DS, Briggs SP, Chamberlin MA, Blakeslee JJ, Murphy AS, Johal GS. Loss of an MDR transporter in compact stalks of maize br2 and sorghum dw3 mutants. Science. 2003:302(5642):81-4.

33. Xing A, Gao Y, Ye L, Zhang W, Cai L, Ching A, Llaca V, Johnson B, Liu L, Yang X, Kang D, Yan J, Li J. A rare SNP mutation in Brachytic2 moderately reduces plant height and increases yield potential in maize. J Exp Bot. 2015; 66(13):3791-802.

34. Knöller A, Blakeslee J, Richards E, Peer W, Murphy A. Brachytic2/ZmABCB1 functions in IAA export from intercalary meristems. J Exp Bot. 2010;61(13): 3689-96.

35. Geisler M, Blakeslee J, Bouchard R, et al. Cellular efflux of auxin catalyzed by the Arabidopsis MDR/PGP transporter AtPGP1. Plant J. 2005;44(2):179-94.

36. Santelia D, Vincenzetti $V$, Azzarello E, Bovet L, Fukao $Y$, Düchtig P, Mancuso S, Martinoia E, Geisler M. MDR-like ABC transporter AtPGP4 is involved in auxin-mediated lateral root and root hair development. FEBS Lett. 2005; 579(24):5399-406.

37. Greene J, Brown N, DiDomenico B, Kaplan J, Eide D. The GEF1 gene of saccharomyces cerevisiae encodes an integral membrane protein; mutations in which have effects on respiration and iron-limited growth. Mol Gen Genet. 1993:241(5-6):542-53.

38. Petrásek J, Friml J. Auxin transport routes in plant development. Development. 2009:136(16):2675-88.

39. Wei L, Zhang $X$, Zhang Z, Liu H, Lin Z. A new allele of the Brachytic2 gene in maize can efficiently modify plant architecture. Heredity. 2018;121(1):75.

40. Balzan S, Carraro N, Salleres B, Cortivo CD, Tuinstra MR, Johal G, Varotto S. Genetic and phenotypic characterization of a novel brachytic2 allele of maize. Plant Growth Regul. 2018;86(1):81-92.

41. Li C, Zheng L, Zhang J, LV Y, Liu J, Wang X, Palfalvi G, Wang G, Zhang Y. Characterization and functional analysis of four $\mathrm{HYH}$ splicing variants in Arabidopsis hypocotyl elongation. Gene. 2017:619:44-9.

42. Geisler M, Murphy A. The ABC of auxin transport: the role of p-glycoproteins in plant development. FEBS Lett. 2006;580(4):1094-102.

43. Hellsberg E, Montanari F, Ecker G. The ABC of phytohormone translocation. Planta Med. 2015;81(6):474-87.

44. Pighin J, Zheng H, Balakshin L, Goodman I, Western T, Jetter R, Kunst L, Samuels A. Plant cuticular lipid export requires an $A B C$ transporter. Science. 2004:306(5696):702-4

45. Nishimura T, Mori Y, Furukawa T, Kadota A, Koshiba T. Red light causes a reduction in IAA levels at the apical tip by inhibiting de novo biosynthesis from tryptophan in maize coleoptiles. Planta. 2006;224(6):1427-35

46. Nishimura T, Koshiba T. Auxin biosynthesis site and polar transport in maize coleoptiles. Plant Signal Behav. 2010;5(5):573-5.

47. Grunewald W, Friml J. The march of the PINs: developmental plasticity by dynamic polar targeting in plant cells. EMBO J. 2010;29(16):2700-14.

48. Mravec J, Kubes M, Bielach A Gaykova V, Petrásek J, Skůpa P, Chand S, Benková E, Zazímalová E, Friml J. Interaction of PIN and PGP transport 
mechanisms in auxin distribution-dependent development. Development. 2008;135(20):3345-54.

49. Bandyopadhyay A, Blakeslee J, Lee O, Mravec J, Sauer M, Titapiwatanakun B, Makam S, Bouchard R, Geisler M, Martinoia E, Friml J, Peer W, Murphy A. Interactions of PIN and PGP auxin transport mechanisms. Biochem Soc Trans. 2007;35(1):137-41.

50. Blakeslee J, Bandyopadhyay A, Lee O, et al. Interactions among PIN-formed and P-glycoprotein auxin transporters in Arabidopsis. Plant Cell. 2007;19(1): 131-47.

51. Sidler M, Hassa P, Hasan S, Ringli C, Dudler R. Involvement of an ABC transporter in a developmental pathway regulating hypocotyl cell elongation in the light. Plant Cell. 2008;10(10):1623-36.

52. Noh B, Murphy A, Spalding E. Multidrug resistance-like genes of Arabidopsis required for auxin transport and auxin-mediated development. Plant Cell. 2001:13(11):2441-54.

53. Weijers D, Schlereth A, Ehrismann J, Schwank G, Kientz M, Jürgens G. Auxin triggers transient local signaling for cell specification in Arabidopsis embryogenesis. Dev Cell. 2006;10(2):265-70.

54. Schenck D, Christian M, Jones A, Lüthen H. Rapid auxin-induced cell expansion and gene expression: a four-decade-old question revisited. Plant Physiol. 2010;152(3):1183-5.

55. Barbier F, Dun E, Beveridge C. Apical dominance. Curr Biol. 2017;27(17):8645 .

56. Kebrom T. A growing stem inhibits bud outgrowth-the overlooked theory of apical dominance. Front Plant Sci. 2017;8:1874.

57. Wang Q, Kohlen W, Rossmann S, Vernoux T, Theres K. Auxin depletion from the leaf axil conditions competence for axillary meristem formation in Arabidopsis and tomato. Plant Cell. 2014a;26(5):2068-79.

58. Wang Y, Wang J, Shi B, Yu T, Qi J, Meyerowitz E, Jiao Y. The stem cell niche in leaf axils is established by auxin and cytokinin in Arabidopsis. Plant Cell. 2014b;26(5):2055-67.

59. Wang Y, Jiao Y. Auxin and above-ground meristems. J Exp Bot. 2018;69(2): 147-54.

60. Tsuda K, Abraham-Juarez M, Maeno A, Dong Z, Aromdee D, Meeley R, Shiroishi T, Nonomura K, Hake S. KNOTTED1 cofactors, BLH12 and BLH14, regulate internode patterning and vein anastomosis in maize. Plant Cell. 2017:29(5):1105-18.

61. Sato-Izawa K, Nakaba S, Tamura K, Yamagishi Y, Nakano Y, Nishikubo N, Kawai S, Kajita S, Ashikari M, Funada R, Katayama Y, Kitano H. DWARF50 (D50), a rice (Oryza sativa L.) gene encoding inositol polyphosphate 5phosphatase, is required for proper development of intercalary meristem. Plant Cell Environ. 2012;35(11):2031-44.

62. Biedroń M, Banasiak A. Auxin-mediated regulation of vascular patterning in Arabidopsis thaliana leaves. Plant Cell Rep. 2018:3(9):1215-29.

63. Long J, Barton M. The development of apical embryonic pattern in Arabidopsis. Development. 1998;125(16):3027-35.

\section{Publisher's Note}

Springer Nature remains neutral with regard to jurisdictional claims in published maps and institutional affiliations.

Ready to submit your research? Choose BMC and benefit from:

- fast, convenient online submission

- thorough peer review by experienced researchers in your field

- rapid publication on acceptance

- support for research data, including large and complex data types

- gold Open Access which fosters wider collaboration and increased citations

- maximum visibility for your research: over $100 \mathrm{M}$ website views per year

At $\mathrm{BMC}$, research is always in progress.

Learn more biomedcentral.com/submissions 